\section{ORIGINAL} RESEARCH

Z. Chu

E.A. Wilde

J.V. Hunter

S.R. McCauley

E.D. Bigler

M. Troyanskaya

R. Yallampalli

J.M. Chia

H.S. Levin

\title{
Voxel-Based Analysis of Diffusion Tensor Imaging in Mild Traumatic Brain Injury in Adolescents
}

BACKGROUND AND PURPOSE: DTI of normal-appearing WM as evaluated by conventional MR imaging in $\mathrm{mTBI}$ has the potential to identify important regional abnormalities that relate to PCS. VBA was used to examine WM changes in acute $\mathrm{mTBI}$.

MATERIALS AND METHODS: WM was assessed between 1 and 6 days postinjury with voxel-based DTI analyses in 10 adolescent patients with $\mathrm{mTBI}$ and 10 age-matched control participants. In addition to the voxel-based group, analysis used to identify brain pathology across all patients with mTBI, 2 voxel-based linear regressions were performed. These analyses investigated the relation between 1) the ADC and PCS severity scores, and 2) ADC and scores on the BSI of emotional symptoms associated with $\mathrm{mTBI}$. We hypothesized that frontotemporal WM changes would relate to symptoms associated with PCS and endorsed on the BSI.

RESULTS: Patients with mTBI demonstrated significant reductions in ADC in several WM regions and in the left thalamus. As expected, no increases in ADC were found in any region of interest. All injury-affected regions showed decreased radial diffusivity, unchanged $A D$, and increased FA, which is consistent with axonal cytotoxic edema, reflective of acute injury.

CONCLUSIONS: Whole-brain WM DTI measures can detect abnormalities in acute mTBI associated with PCS symptoms in adolescents.

\begin{abstract}
ABBREVIATIONS: $A D=$ axial diffusivity; $A D C=$ apparent diffusion coefficient; $B d b=$ slope $10^{-9}$ $\mathrm{mm}^{2} / \mathrm{sec} / \mathrm{BSI} ; \mathrm{Bdp}=$ slope $10^{-9} \mathrm{~mm}^{2} / \mathrm{sec} / \mathrm{RPCSQ} ; \mathrm{Bfb}=$ slope $10^{-3} 1 / \mathrm{BSI} ; \mathrm{Bfp}=$ slope $10^{-3}$ 1/RPCSO; $\mathrm{BSI}=$ Brief Symptom Inventory; $\mathrm{Ca}=$ calcium; $\mathrm{CC}=$ corpus callosum; $\mathrm{DTI}=$ diffusion tensor imaging; $F A=$ fractional anisotropy; $\mathrm{GM}=$ gray matter; $\mathrm{GRP}=$ group; $\mathrm{L}=$ left; $\mathrm{mTBI}=$ mild traumatic brain injury; $\mathrm{NA}=$ no statistical values; $\mathrm{MNI}=$ Montreal Neurological Institute; PCS = postconcussive symptoms; $r=$ Pearson product-moment correlation coefficient; $\mathrm{R}=$ right; $\mathrm{RD}=$ radial diffusivity; RPCSQ = Rivermead Post Concussion Symptoms Questionnaire; SENSE = sensitivity encoding; SPM = statistical parametric mapping; $\mathrm{TBI}=$ traumatic brain injury; VBA = voxel-based analysis; $\mathrm{WM}=$ white matter
\end{abstract}

A MR imaging methods have improved, the detection of abnormalities associated with TBI has become increasingly more sensitive. This is an especially important issue in mTBI because conventional MR imaging most often demonstrates what appears to be normal-appearing WM. However, DTI and other advanced MR imaging methods, such as susceptibility-weighted imaging, offer better detection of traumarelated injury occurring from shearing forces within brain pa-

Received May 4, 2009; accepted after revision June 24.

From the E.B. Singleton Department of Diagnostic Imaging (Z.C., E.A.W., J.V.H.), Texas Children's Hospital, Houston, Texas; Department of Radiology (Z.C., E.A.W., J.V.H.), Baylor College of Medicine, Houston, Texas; Physical Medicine and Rehabilitation Alliance (E.A.W., S.R.M., M.T., R.Y., H.S.L.), Baylor College of Medicine and University of TexasHouston Medical School, Houston, Texas; Departments of Psychology and Neuroscience (E.D.B.), Brigham Young University, Provo, Utah; Department of Psychiatry and Brain Institute (E.D.B.), University of Utah, Salt Lake City, Utah; and Philips Medical Systems (J.M.C.), Cleveland, Ohio.

This work was supported by grant P01 NS056202 from the National Institutes of Neurological Disorders and Stroke and grant R01 NS21889 from the National Institutes of Child Health and Human Development.

Previously presented in part at: 16th Scientific Meeting and Exhibition of the International Society for Magnetic Resonance in Medicine, May 3-9. 2009; Toronto, Ontario, Canada.

Please address correspondence to Zili Chu, PhD, E.B. Singleton Department of Diagnostic Imaging, Texas Children's Hospital, 6621 Fannin St, MC 2-2521, Houston, TX 77030; e-mail: zxchu@texaschildrens.org

Indicates open access to non-subscribers at www.ajnr.org

indicates article with supplemental on-line tables.

DOI 10.3174/ajnr.A1806 renchyma, and they have demonstrated a variety of consistent abnormalities across a number of mTBI studies. ${ }^{1-7}$

Detailed biomechanical and heuristics studies consistently show the vulnerability of WM, particularly the CC and longcoursing fasciculi, especially within frontotemporal regions. ${ }^{8-12}$ DTI abnormalities within the CC in patients with TBI have been well documented in both the acute and chronic phase of injury. ${ }^{13-18}$ Because of the ease with which the CC can be delineated by MR imaging as a region of interest along with being the largest commissural fiber tract in the brain, the CC is also the most widely investigated WM brain structure in TBI. ${ }^{10,19}$

In a previous DTI studies of the CC in acute mTBI in adolescent patients, Wilde et $\mathrm{al}^{17}$ found distinct DTI differences in the injured group. More important, findings were related to PCS indicating that DTI metrics not only have the potential to reveal neuropathologic changes associated with trauma but can clinically relate to the acute status of the patient who has a brain injury. During the acute phase of brain injury, changes occur in the WM that affect the free dispersion of water and hence the DTI signal intensity. Thus, DTI findings in acute mTBI differ from what is typically seen in more chronic TBI, wherein DTI-defined WM abnormalities reflect degenerative changes. ${ }^{20}$

Lipton et $\mathrm{al}^{4}$ have shown that in adults with mTBI and lasting cognitive sequelae, nonspecific WM changes were observed that were similar to those in reports of nonspecific sub- 


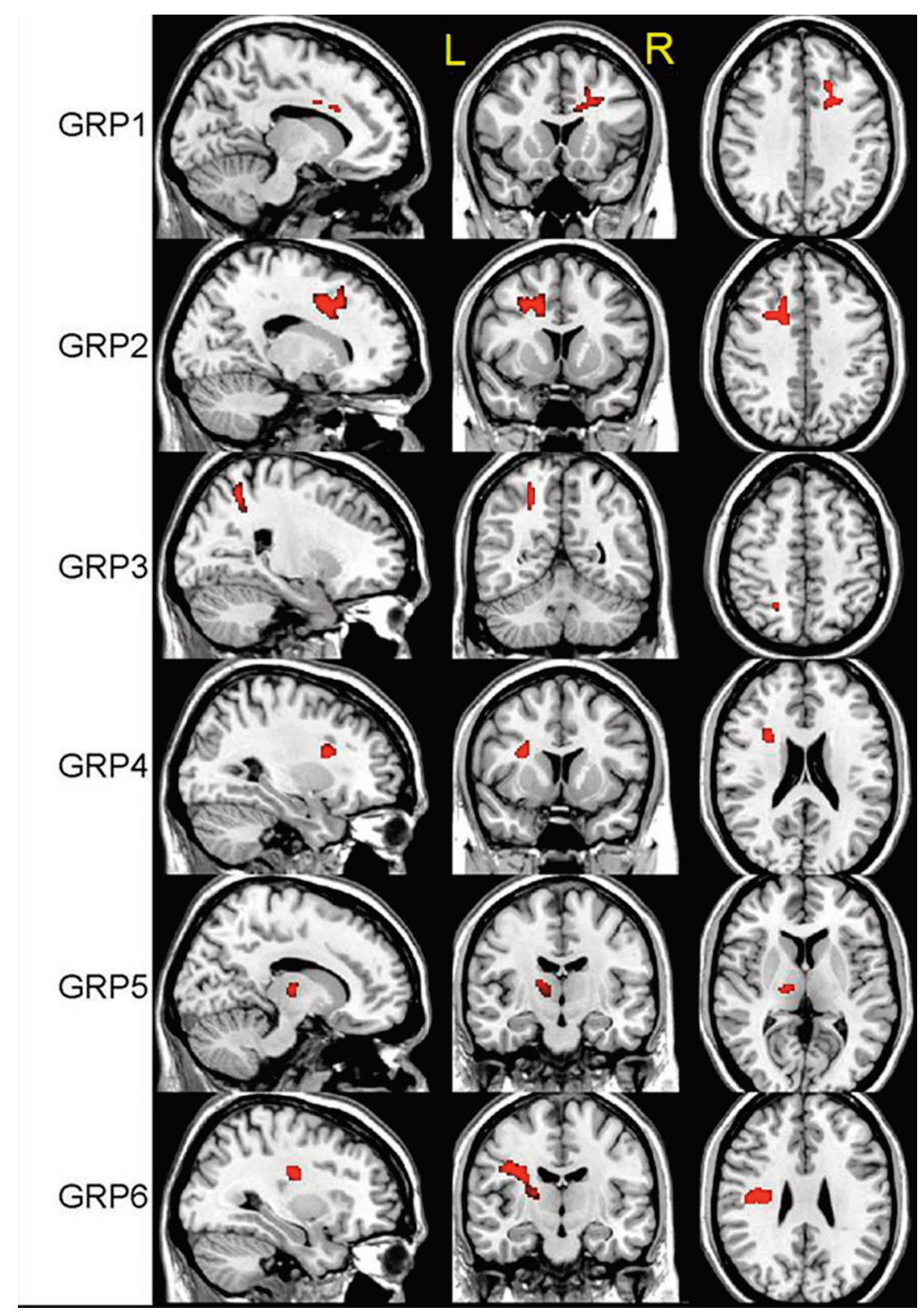

Fig 1. Three-plane views of 6 regions (GRP 1-6) identified by ADC-GRP analysis, superimposed on a structural image.

cortical WM changes in more severe TBI but to a lesser degree. Nonetheless, the detected chronic WM lesions associated with mTBI were widespread. Given these previous DTI findings, we hypothesized that DTI metrics of WM integrity would also show widespread changes during the acute phase of $\mathrm{mTBI}$ in adolescents. We also predicted that changes in extracallosal WM would be similar to those observed in the CC, likely reflecting common regions of acute but localized neuroinflammation. ${ }^{21}$

Detecting WM abnormalities outside a well-defined structure like the CC benefits from methods that compare voxelby-voxel DTI characteristics of the whole brain. ${ }^{13,16}$ Such whole-brain DTI analyses have frequently been applied to the study of severe TBI in adults, ${ }^{15,22,23}$ though some have addressed pediatric TBI as well, ${ }^{18,20}$ but mostly in the chronic phase. In the acute phase, DTI metrics are taking advantage of the movement of water that is held intra- and extracellularly and within the myelin, where swelling from localized neuroinflammation restricts water dispersion. Following TBI, the free space between neighboring swollen axonal bundles is reduced, thereby resulting in decreased $\mathrm{RD}^{24}$ while leaving longitudinal diffusivity (or AD) unchanged. The frequently used indices in DTI analysis are FA and ADC. However, it has been proposed that FA and ADC are not sufficient to explain all aspects of pathologic change because of the complexity of the structure. Therefore, $\mathrm{RD}$, which reflects the diffusivity perpendicular to the longitudinal axis of the axon and is calculated as the mean of the secondary and tertiary eigenvalues, ${ }^{25}$ has been introduced in addition to FA and ADC.

A limitation of the Wilde et al study ${ }^{17}$ was that it did not examine noncallosal WM, and the current investigation extends their original findings by applying whole-brain DTI analyses to acutely injured patients with mTBI. We hypothesized that voxel-based whole-brain WM DTI analyses in 


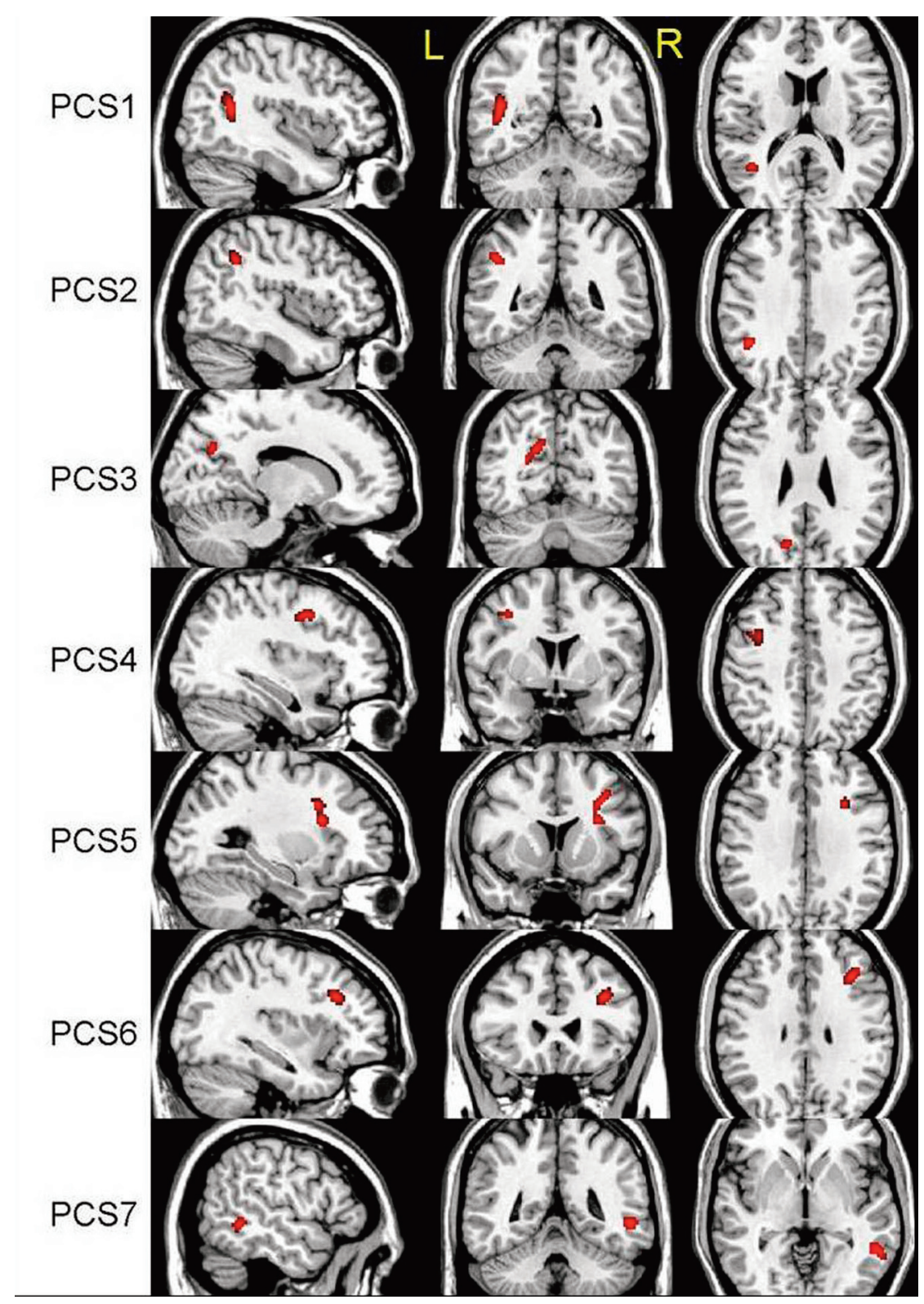

Fig 2. Three-plane views of 7 regions (PCS 1-7) identified by ADC-RPCSO analysis, superimposed on a structural image

the acute injury phase of mTBI would demonstrate reduced ADC, similar to that observed in the CC, particularly in frontotemporal WM regions. Furthermore, because symptoms associated with PCS and affective changes are commonplace in acute $\mathrm{mTBI},{ }^{26}$ we postulated that acute DTI findings in mTBI would correlate with clinical mTBI sequelae.

\section{Methods and Materials}

\section{Participants}

The study consisted of 10 right-handed patients with mTBI (4 males, 6 females; mean age, $15.70 \pm 1.18$ years; range, $14-17$; education, $9.60 \pm 1.71$ years with the range of $7-12$ years) and 10 demographically matched right-handed control participants (4 males, 6 females; mean age, $15.70 \pm 1.83$ years; range, $14-19$; education, $9.90 \pm 1.85$ years with a range of $8-12$ years). The initial Glasgow Coma Scale ${ }^{27}$ score was 15 for all patients with mTBI. The postinjury intervals for scanning and testing were 2.7 days (range, 1-6 days) and 3.4 days (range, 1-6 days), respectively. All patients with mTBI included in this study had no positive findings on head CT performed within 24 hours after injury. All participants including the mTBI and control groups had no preinjury history of neurologic or psychiatric disorders. This research protocol was approved by the institutional review board of the Baylor College of Medicine. Informed consent was obtained from each participant's guardian or parent before the study, and consent was provided by the participants.

\section{Measures}

The severity of PCS and level of general emotional distress were measured by using participant reports from the RPCSQ ${ }^{28}$ and the Global Severity Index from the BSI, ${ }^{29}$ respectively. The RPCSQ consists of 3 subscales ${ }^{30}$ including indices of cognitive (eg, memory and concentration difficulties), emotional (eg, anxiety, restlessness, and depression), and somatic (eg, fatigue, headaches, dizziness, nausea, sleep 


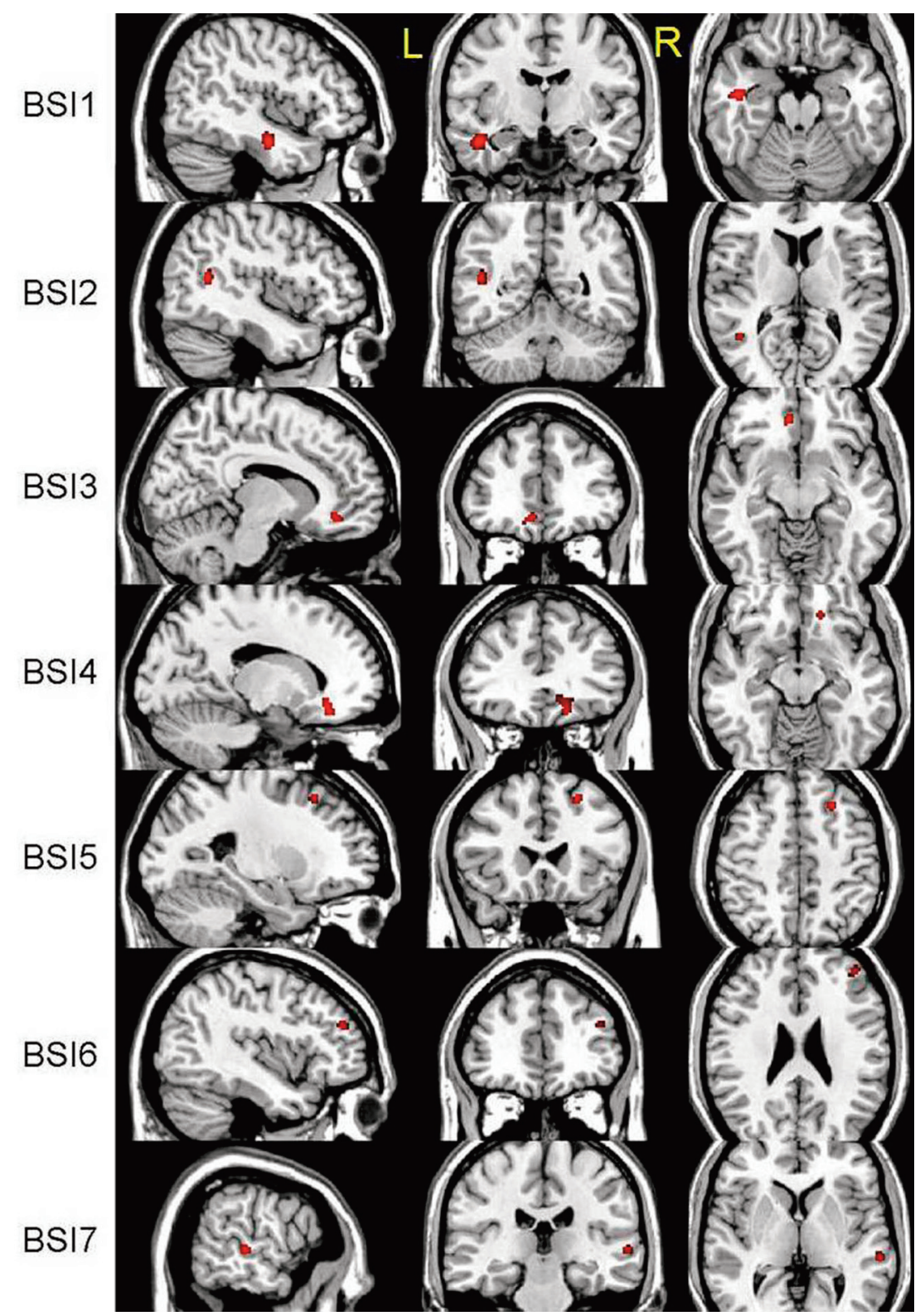

Fig 3. Three-plane views of 7 regions (BSI 1-7) identified by ADC-BSI analysis, superimposed on a structural image.

disturbance, and changes in vision) symptoms commonly following mTBI. The Global Severity Index from the BSI is a measure of general emotional functioning, which encompasses multiple areas of psychiatric symptoms (eg, affective and somatic domains). Raw scores from the RPCSQ and the T-score from the BSI were used, with higher scores indicating greater symptom severity.

\section{Imaging Protocols}

In all cases, the CT and MR images were read by a board-certified neuroradiologist (J.V.H). The MR imaging data were acquired by using a $3 \mathrm{~T}$ Achieva scanner (Philips Medical Systems, Best, the Netherlands) with a SENSE 8-channel head coil. In addition to conventional MR images (including diffusion-weighted and susceptibility-weighted imaging, which did not reveal abnormalities in any participants except 1 -see Wilde et $\mathrm{al}^{17}$ ), DTI was performed to measure the integrity of WM (axial single-shot spinecho echo-planar imaging sequence with 30 diffusion-encoding directions; 224-mm FOV; acquisition voxel size, $2 \times 2 \times 2 \mathrm{~mm}^{3}$;
TR, $6318.0 \mathrm{~ms}$; TE, $51 \mathrm{~ms}$; SENSE reduction factor of 2; $2 \mathrm{~b}$ factors with $0 \mathrm{~s} / \mathrm{mm}^{2}$, low $\mathrm{b}$, and $1000 \mathrm{~s} / \mathrm{mm}^{2}$, high b), with 2 acquisitions for averaging.

\section{MR Imaging Postprocessing}

The DTI registration tool (Release 0.4; Philips Medical Systems) for PRIDE V4 was used to remove motion and eddy current artifacts and also to average 2 acquisitions for better signal intensity-to-noise ratio. The averaged DTI data were loaded into DTIStudio (Version 2.40, Johns Hopkins University, Baltimore, Maryland; http://www.fil.ion. ucl.ac.uk) to compute and export DTI index maps, including 3 eigenvalues, ADC, and FA. The secondary and tertiary eigenvalue maps were averaged to create the $\mathrm{RD}$ map. In addition, $b=0$ images were also exported to be used as the reference image in creating a template image of the FA map in MNI space. All DTI index maps were subsequently normalized to the template and then smoothed with a gaussian filter of full width at half maximum of $8 \mathrm{~mm}$ to minimize the effect of partial volume and registration error during statistical anal- 
yses. The normalized $b=0$ image was segmented by using the SPM2 software (Wellcome Department of Imaging Neuroscience, London, UK) segmentation routine to yield 3 tissue masks: WM, GM, and CSF, which were used in regional analyses of whole-brain tissues. After data preparation, the VBA was performed by using SPM2.

\section{Voxel-Based Statistical Analysis}

The Student unpaired $t$ test in SPM2 was performed between patient and control groups to evaluate ADC differences (ADC-GRP analysis). In addition, linear regression was used to investigate the respective relation between the following 2 pairs of variables: 1) ADC and PCS scores (ADC-RPCSQ analysis), and 2) ADC and BSI scores (ADC-BSI analysis). The whole-brain mask was used to exclude anything outside the brain. Because the patients in this study sustained relatively mild TBI, it was expected that the injury-related differences in GM would be small in comparison with those in the WM. Moreover, no intrinsic difference was anticipated in CSF, which renders it an ideal region to use as a baseline measure for noise. In the present study, both ADC increases and decreases were examined in probabilistic maps to extract any statistically significant regions. All significant regions were exported by using MarsBaR (MARSeille Boîte À Région d'Intérêt; http://sourceforge.net/projects/marsbar/) toolbox for $\mathrm{SPM}^{31}$ as regions used subsequently in a refined region-based statistical analysis.

\section{Region-Based Statistical Analysis}

All region-based analyses were performed on the mean value, which is the average over 1 targeted region in the unsmoothed DTI index map. This region-based analysis was extended to all DTI indices, including FA, RD, and ADC. Region-based statistical analyses were performed by using the Statistical Package for the Social Sciences for Windows, Version 15.0 (SPSS, Chicago, Illinois). The composite region may provide information related to general vulnerability despite interindividual variation in the mechanism and degree of injury. On the basis of this assumption, the region-based analysis was also performed on composite regions. Furthermore, the region-based analysis was also applied to the whole CSF in assessing the baseline DTI index maps as well as to the whole GM and WM.

For display purposes, regions identified through statistical analysis were superimposed on a structural MR image in MNI space. To localize and label brain structures, we transferred the MNI coordinates into the Talairach space ${ }^{32}$ by using Talairach Daemon (http://www.nitrc.org/docman/?group_id=54).

\section{Results}

VBA revealed 6 regions with significant decreases in ADC, $t(18)=3.6, P<.001$, by using a cluster size of $>50$ voxels (Fig 1). As expected, most regions identified were located in WM structures with the exception of the left thalamus. No region was found to have a significant increase in ADC by using the same thresholds. The linear regression analysis between ADC and RPCSQ indicated a significant negative relation in 7 regions, $t(18)=3.3, P<.002$, by using a cluster size of $>40$ voxels (Fig 2), which were again located predominantly in the WM. Again, no significant regions between ADC increase and RPCSQ were identified, consistent with the results of the group analysis.

Linear regression analysis of the relation between $\mathrm{ADC}$ and BSI revealed 7 regions that were negatively related by using the thresholds above. No positive relations were found (Fig 3). Twenty regions of significant difference were identified by

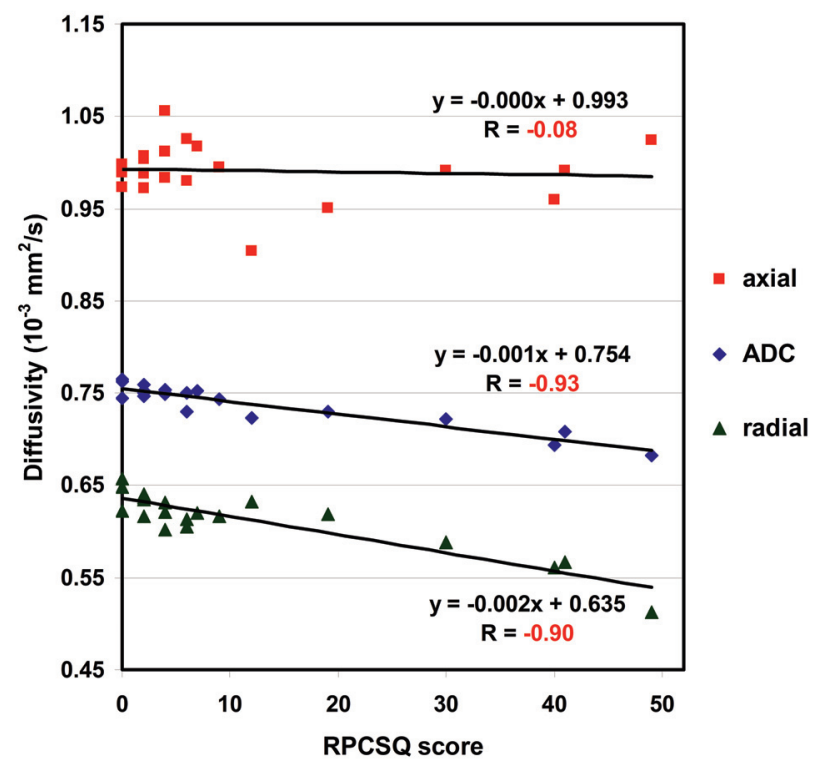

Fig 4. Scatterplot with regression lines demonstrating the linear correlation of RPCSO and diffusivities, including $A D C, A D$, and $R D$, respectively, on the combined regions.

VBA, (ie, 6 regions identified by group analysis of ADC only; 7, by analysis of ADC-RPCSQ; and 7, by analysis of ADC-BSI). Additionally, 3 composite regions were created on the basis of the results of these 3 analyses. Therefore, there were a total of 23 regions for the following region-based analyses.

The region-based analysis was subsequently performed on all DTI index maps to investigate group difference and the relationship to RPCSQ and BSI in each of those 23 regions. Consistent with the voxel-based analyses, all regions showed negative relations between RPCSQ and ADC and RD, suggesting that diffusivity decreased as the RPCSQ score increased. In contrast, FA demonstrated a positive relation to RPCSQ, suggesting that FA increased as the RPCSQ score increased. Moreover, linear regression analysis on the combined region showed the same relation as that of each individual region, but with a stronger correlation. Figure 4 shows the relation between ADC and RPCSQ by using linear regression on the first combined region.

The on-line Tables illustrate the highly significant group differences for the composite regions for ADC, $t(18)=-7.14$, $P<.001, \mathrm{RD}, t(18)=-7.08, P<.001$, and FA, $t(18)=4.03$, $P<.001$. No significant change for $\mathrm{AD}, t(18)=-1.72, P<$ .102 , was noted. Additionally, the composite regions demonstrated highly significant correlations between RPCSQ scores and ADC decrease $(r=-0.94, P<.001)$, RD decrease $(r=$ $-0.90, P<.001)$, and FA increase $(\mathrm{r}=0.69, P=.001)$ in the mTBI group. No correlation was observed between RPCSQ score and AD change $(r=-0.08, P=.742)$ in the mTBI group. Finally, the composite region from the ADC-BSI analysis also demonstrated a highly significant correlation between the BSI score and ADC $(r=-0.91, P=.001)$ and RD decrease $(r=-0.88, P=.001)$, and $\mathrm{AD}(r=-0.45)$ and FA change $(r=-0.64, P=.001)$ in the mTBI group. Region-based analyses including testing for group differences and the relation of each region to RPCSQ and BSI were applied to all 23 regions as cross analyses. The cross analyses demonstrated that the relation between both the ADC and RPCSQ and between ADC 


\begin{tabular}{|c|c|c|c|c|c|c|c|c|}
\hline \multirow[b]{2}{*}{ Regions } & \multicolumn{2}{|c|}{$A D C$} & \multicolumn{2}{|c|}{ FA } & \multicolumn{2}{|c|}{$A D$} & \multicolumn{2}{|c|}{$\mathrm{RD}$} \\
\hline & $t$ & $P$ Value & $t$ & $P$ Value & $t$ & $P$ Value & $t$ & $P$ Value \\
\hline GM & -0.99 & .338 & NA & NA & -0.37 & .715 & -1.26 & .223 \\
\hline WM & -2.45 & .025 & 1.70 & 0.107 & -1.74 & .099 & -2.17 & .044 \\
\hline
\end{tabular}

a Unpaired $t$ tests between the patients and the controls were used on whole GM and WM. The $P$ values have been corrected for multiple dependent comparisons.

\begin{tabular}{|c|c|c|c|c|c|c|c|c|}
\hline \multirow[b]{2}{*}{ Regions } & \multicolumn{2}{|c|}{$A D C$} & \multicolumn{2}{|c|}{ FA } & \multicolumn{2}{|c|}{$A D$} & \multicolumn{2}{|c|}{$\mathrm{RD}$} \\
\hline & $r$ & Bdp & $r$ & Bfp & $r$ & Bdp & $r$ & Bdp \\
\hline \multicolumn{9}{|c|}{ ADC-RPCSQ correlation } \\
\hline GM & -0.295 & -0.536 & NA & NA & -0.107 & -0.191 & -0.375 & -0.705 \\
\hline WM & -0.460 & -0.422 & 0.589 & 0.562 & -0.053 & -0.052 & -0.548 & -0.604 \\
\hline \multicolumn{9}{|c|}{ ADC-BSI correlation } \\
\hline GM & -0.093 & -0.310 & NA & NA & 0.073 & 0.240 & -0.168 & -0.581 \\
\hline WM & -0.356 & -0.601 & 0.489 & 0.859 & 0.019 & 0.003 & -0.453 & -0.916 \\
\hline
\end{tabular}

a The correlation between ADC and test scores (RPCSO and BSI, respectively) on whole GM and WM, where NA indicates no statistical values since FA on GM regions is less than threshold (0.21).

and BSI has similarly highly significant relations on regions that were identified by either ADC-RPCSQ or ADC-BSI linear regression analysis methods, even when these regions did not significantly differ by group for ADC only.

The analysis of the relation between diffusivity and RPCSQ revealed that diffusivity along the axonal direction $(\mathrm{AD})$ was not significantly changed in the patients with mTBI, while the correspondent diffusivity perpendicular to the axonal direction (RD) decreased significantly in a number of regions. This was consistent with the observed decrease in ADC and increase in FA in those regions.

Statistical analyses on segmentation of GM, WM, and CSF (Tables 1 and 2) revealed no significant group difference on CSF or GM. By contrast, results showed a moderate ADC decrease and an increase in FA in WM, suggesting that the injury-affected area in WM is significant, presumably covering most WM, consistent with the diffuse nature of diffuse axonal injury in TBI.

\section{Discussion}

As expected, all participants had normal-appearing WM on $\mathrm{CT}$, and all except 1 had normal-appearing WM on conventional MR imaging. VBA revealed significant differences between groups, with increased FA and decreased ADC, evident in multiple brain regions in the mTBI group. Although FA is often found to be reduced in chronic mTBI, reflecting disrupted integrity of WM fiber bundles with accompanying freer water dispersion due to cellular and axonal damage, ${ }^{6}$ increased FA and decreased ADC in acute injury may result from cytotoxic edema and localized inflammatory responses. The results obtained in the current study by using VBA are consistent with recent reports of increased FA and decreased ADC in acute brain injury. ${ }^{17,33}$

In the original mTBI study by Wilde et $\mathrm{al},{ }^{17}$ decreased ADC and increased FA were found within the midsagittal fibers traversing the $\mathrm{CC}$ in patients with acute $\mathrm{mTBI}$ relative to controls. As expected, VBA revealed similarly decreased ADC in regions with projections through the CC but also demonstrated several additional areas of decreased ADC (Fig 1). Even with diverse causes of injury, it appears that there are areas of commonality of both WM and GM regions in mTBI. As with more severe injury, there appear to be diffuse changes in WM regions with known susceptibility to shear-strain forces, such as the superior frontal longitudinal fasciculus and the cingulate bundle. In addition, change in association regions such as the thalamus may not be surprising, given the complex connectivity to other regions. Findings suggest that the effects of mTBI are indeed widespread, consistent with the distribution of DTI abnormalities observed during the chronic phase following mTBI. ${ }^{4-6,34}$ Given that the participants were all scanned within 6 days of injury, it is plausible that some of these acute abnormalities may evolve into more chronic WM changes. Longitudinal DTI investigation of patients with mTBI is underway to evaluate this further.

We previously suggested that the DTI changes observed in acute mTBI were likely attributable to cytotoxic edema in this initial postinjury interval, and in the current study, we performed further detailed examination of $\mathrm{AD}$ in addition to $\mathrm{FA}$, $\mathrm{ADC}$, and $\mathrm{RD}$. The lack of change in $\mathrm{AD}$ combined with the significant drop in RD is consistent with axonal swelling. Acceleration and deceleration forces have been previously shown to cause defects of myelin lamellae and axolemmas, consequently leading to axonal swelling with the release of large amounts of $\mathrm{Ca}^{2+} \cdot 35,36$ Such axonal swelling may reduce the free space of water, thus reducing diffusivity.

The relation of DTI parameters including additional analyses examining the relation between ADC and RPCSQ and between $\mathrm{ADC}$ and $\mathrm{BSI}$ demonstrated several regions with significant decreases in ADC in patients with mTBI. Not unexpectedly, $\mathrm{ADC}$ was related to the BSI in regions identified by ADC-RPCSQ analysis, and ADC was also correlated with RPCSQ in regions identified by ADC-BSI, owing to the high correlation between RPCSQ and BSI $(r=0.62)$. All of this suggests that perturbation of WM and its interconnectivity resulting from the neuropathologic effects of mTBI may be associated with PCS symptoms. It may be that early identification of pathologic brain changes in mTBI will aid in the identification and classification of the disorder, which is likely underdiagnosed due to lack of specific medical biomarkers to date $\mathrm{e}^{37}$ and in turn may lead to earlier and more effective interventions. ${ }^{38,39}$ 


\section{Conclusions}

The present study revealed significant alteration in DTI metrics in a group of patients with $\mathrm{mTBI}$ in several brain regions, and these changes were highly correlated with PCS severity and emotional distress. Detailed analyses of the diffusivities along different directions demonstrate the likelihood of cytotoxic edema as the probable mechanism of injury in acute mTBI. Voxel-based DTI analysis is capable of identifying potentially diffuse axonal injury-vulnerable regions invisible to $\mathrm{CT}$ and conventional MR imaging, which may assist in classification, early diagnosis, and treatment.

\section{References}

1. Kraus MF, Susmaras T, Caughlin BP, et al. White matter integrity and cognition in chronic traumatic brain injury: a diffusion tensor imaging study. Brain 2007;130:2508-19

2. Lee $\mathrm{H}$, Wintermark $\mathrm{M}$, Gean $\mathrm{AD}$, et al. Focal lesions in acute mild traumatic brain injury and neurocognitive outcome: CT versus 3T MRI. J Neurotrauma 2008;25:1049-56

3. Levine B, Kovacevic N, Nica EI, et al. The Toronto traumatic brain injury study: injury severity and quantified MRI. Neurology 2008;70:771-78

4. Lipton ML, Gellella E, Lo C, et al. Multifocal white matter ultrastructural abnormalities in mild traumatic brain injury with cognitive disability: a voxel-wise analysis of diffusion tensor imaging. J Neurotrauma 2008;25:1335-42

5. Niogi SN, Mukherjee P, Ghajar J, et al. Extent of microstructural white matter injury in postconcussive syndrome correlates with impaired cognitive reaction time: a 3T diffusion tensor imaging study of mild traumatic brain injury. AJNR Am J Neuroradiol 2008;29:967-73

6. Rutgers DR, Toulgoat F, Cazejust J, et al. White matter abnormalities in mild traumatic brain injury: a diffusion tensor imaging study. AJNR Am J Neuroradiol 2008;29:514-19

7. Xu J, Rasmussen IA, Lagopoulos J, et al. Diffuse axonal injury in severe traumatic brain injury visualized using high-resolution diffusion tensor imaging. J Neurotrauma 2007;24:753-65

8. Bayly PV, Black EE, Pedersen RC, et al. In vivo imaging of rapid deformation and strain in an animal model of traumatic brain injury. $J$ Biomech 2006;39:1086-95

9. Bayly PV, Ji S, Song SK, et al. Measurement of strain in physical models of brain injury: a method based on HARP analysis of tagged magnetic resonance images (MRI). J Biomech Eng 2004;126:523-28

10. Sabet AA, Christoforou E, Zatlin B, et al. Deformation of the human brain induced by mild angular head acceleration. J Biomech 2008;41:307-15

11. Takhounts EG, Ridella SA, Hasija V, et al. Investigation of traumatic brain injuries using the next generation of simulated injury monitor (SIMon) finite element head model. Stapp Car Crash J 2008;52:1-31

12. Yoganandan N, Li J, Zhang J, et al. Influence of angular acceleration-deceleration pulse shapes on regional brain strains. J Biomech 2008;41:2253-62

13. Bendlin BB, Ries ML, Lazar M, et al. Longitudinal changes in patients with traumatic brain injury assessed with diffusion-tensor and volumetric imaging. Neuroimage 2008;42:503-14

14. Ewing-Cobbs L, Prasad MR, Swank P, et al. Arrested development and disrupted callosal microstructure following pediatric traumatic brain injury: relation to neurobehavioral outcomes. Neuroimage 2008;42:1305-15

15. Kumar R, Husain M, Gupta RK, et al. Serial changes in the white matter diffusion tensor imaging metrics in moderate traumatic brain injury and correlation with neuro-cognitive function. J Neurotrauma 2009;26:481-95
16. Sidaros A, Engberg AW, Sidaros K, et al. Diffusion tensor imaging during recovery from severe traumatic brain injury and relation to clinical outcome: a longitudinal study. Brain 2008;131:559-72

17. Wilde EA, McCauley SR, Hunter JV, et al. Diffusion tensor imaging of acute mild traumatic brain injury in adolescents. Neurology 2008;70:948-55

18. Yuan W, Holland SK, Schmithorst VJ, et al. Diffusion tensor MR imaging reveals persistent white matter alteration after traumatic brain injury experienced during early childhood. AJNR Am J Neuroradiol 2007;28:1919-25

19. Dubroff JG, Newberg A. Neuroimaging of traumatic brain injury. Semin Neurol 2008;28:548-57

20. Levin HS, Wilde EA, Chu Z, et al. Diffusion tensor imaging in relation to cognitive and functional outcome of traumatic brain injury in children. J Head Trauma Rehabil 2008;23:197-208

21. Morganti-Kossmann MC, Satgunaseelan L, Bye N, et al. Modulation of immune response by head injury. Injury 2007;38:1392-400

22. Benson RR, Meda SA, Vasudevan S, et al. Global white matter analysis of diffusion tensor images is predictive of injury severity in traumatic brain injury. J Neurotrauma 2007;24:446-59

23. Tollard E, Galanaud D, Perlbarg V, et al. Experience of diffusion tensor imaging and $1 \mathrm{H}$ spectroscopy for outcome prediction in severe traumatic brain injury: preliminary results. Crit Care Med 2009;37:1448-55

24. Alexander AL, Lee JE, Lazar M, et al. Diffusion tensor imaging of the brain. Neurotherapeutics 2007;4:316-29

25. Song SK, Sun SW, Ju WK, et al. Diffusion tensor imaging detects and differentiates axon and myelin degeneration in mouse optic nerve after retinal ischemia. Neuroimage 2003;20:1714-22

26. Bigler ED. Neuropsychology and clinical neuroscience of persistent post-concussive syndrome. J Int Neuropsychol Soc 2008;14:1-22

27. Teasdale G, Jennett B. Assessment of coma and impaired consciousness: a practical scale. Lancet 1974;2:81-84

28. King NS, Crawford S, Wenden FJ, et al. The Rivermead Post Concussion Symptoms Questionnaire: a measure of symptoms commonly experienced after head injury and its reliability. J Neurol 1995;242:587-92

29. Derogatis LR. Brief Symptom Inventory (BSI): Administration, Scoring, and Procedure Manual. Minneapolis, Minnesota: NCS Pearson; 1993

30. Smith-Seemiller L, Fow NR, Kant R, et al. Presence of post-concussion syndrome symptoms in patients with chronic pain vs mild traumatic brain injury. Brain Inj 2003;17:199-206

31. Brett M, Anton J, Valabregue R, et al. Region of interest analysis using an SPM toolbox. In: Proceedings of the 8th International Conference on Functional Mapping of the Human Brain, Sendai, Japan. June 2-6, 2002

32. Talairach J, Tournoux P. Co-Planar Stereotaxic Atlas of the Human Brain. Stuttgart, Germany: Thieme Verlag; 1988

33. Bazarian JJ, Zhong J, Blyth B, et al. Diffusion tensor imaging detects clinically important axonal damage after mild traumatic brain injury: a pilot study. J Neurotrauma 2007;24:1447-59

34. Niogi SN, Mukherjee P, Ghajar J, et al. Structural dissociation of attentional control and memory in adults with and without mild traumatic brain injury. Brain 2008;131:3209-21

35. Buki A, Povlishock JT. All roads lead to disconnection?-Traumatic axonal injury revisited. Acta Neurochir (Wien) 2006;148:181-93. discussion 193-94. Epub 2005 Dec 20

36. Maxwell WL, Kansagra AM, Graham DI, et al. Freeze-fracture studies of reactive myelinated nerve fibres after diffuse axonal injury. Acta Neuropathol 1988;76:395-406

37. Powell JM, Ferraro JV, Dikmen SS, et al. Accuracy of mild traumatic brain injury diagnosis. Arch Phys Med Rehabil 2008;89:1550-55

38. Casson IR, Pellman EJ, Viano DC. Concussion in the national football league: an overview for neurologists. Neurol Clin 2008;26:217-41, X-xi

39. Mami AG, Nance ML. Management of mild head injury in the pediatric patient. Adv Pediatr 2008;55:385-94 